\title{
Effects of a Medication Review on Delirium in Older Hospitalised Patients: A Comparative Retrospective Cohort Study
}

\author{
Eveline L. van Velthuijsen ${ }^{1}$ - Sandra M. G. Zwakhalen ${ }^{1} \cdot$ Evelien Pijpers $^{2}$ • \\ Liesbeth I. van de Ven $^{3}$ - Ton Ambergen ${ }^{4}$ Wubbo J. Mulder ${ }^{2}$ Frans R. J. Verhey ${ }^{5}$. \\ Gertrudis I. J. M. Kempen ${ }^{1}$
}

Published online: 2 February 2018

(C) The Author(s) 2018. This article is an open access publication

\begin{abstract}
Background Delirium in older hospitalised patients is a common and serious disorder. Polypharmacy and certain medications are risk factors for developing delirium. A medication review could benefit older hospitalised patients with delirium.

Objectives (1) Evaluate the effects of medication review on length of delirium, length of hospital stay, mortality, and discharge destination; and (2) describe and analyse the proposed changes to medication and its implementation by the treating physician.

Setting The study was conducted at Maastricht University Medical Centre+.

Methods We compared two cohorts of older patients with delirium: the first cohort from before introducing the
\end{abstract}

Electronic supplementary material The online version of this article (https://doi.org/10.1007/s40266-018-0523-9) contains supplementary material, which is available to authorized users.

Eveline L. van Velthuijsen

e.vanvelthuijsen@maastrichtuniversity.nl

1 Department of Health Services Research, Care and Public Health Research Institute (CAPHRI), Maastricht University, P.O. Box 616, 6200 MD Maastricht, The Netherlands

2 Department of Internal Medicine, Maastricht University Medical Centre+, Maastricht, The Netherlands

3 Department of Clinical Pharmacy and Toxicology, Maastricht University Medical Centre+, Maastricht, The Netherlands

4 Department of Methodology and Statistics, Maastricht University, Maastricht, The Netherlands

5 Department of Psychiatry and Neuropsychology, Alzheimer Centre Limburg, MHeNS School for Mental Health and NeuroScience, Maastricht University, Maastricht, The Netherlands medication review, and a second cohort 5 months after introduction of the medication review. Data were extracted from the patients' digital medical records.

Results A significant interaction effect of cohort and number of medications taken by the patient was found for duration of delirium: patients from the second cohort taking between zero and six medications had significantly shorter delirious episodes than patients in the first cohort. This effect bordered on significance for patients taking between seven and 11 medications, but disappeared for patients taking 12 or more medications. No other statistically significant differences were found between the cohorts. The proposed changes in medication were implemented for $71 \%$ of the patients.

Conclusion A medication review seems to significantly decrease the length of an older patient's delirious episode. Given the clinical relevance of these findings, we advise medication reviews for all older patients who are delirious or are at risk of developing delirium.

\section{Key Points}

Patients who received a medication review from a clinical pharmacist were likely to have shorter delirious episodes.

Based on the clinical relevance of a shorter delirious episode, the authors advise to provide a medication review for all patients who are suffering delirium or are at risk for developing it.

Physicians implemented most of the changes in medication suggested by the clinical pharmacist, indicating that the advice is a useful tool for physicians to reconcile a patient's medication list. 


\section{Introduction}

Delirium is a serious neuropsychiatric syndrome characterised by an acute decline of cognitive functions and disorientation [1] affecting between 29 and $64 \%$ of older hospitalised patients [2]. It is associated with many negative outcomes, including increased mortality rates, prolonged hospital stay, decreased physical recovery, and higher rates of institutionalisation [3-5]. These negative consequences not only affect patients, but also greatly increase healthcare costs [6-9]. There is substantial evidence suggesting that delirium is preventable in $30-40 \%$ of cases, mainly by avoiding iatrogenic risk factors which could trigger delirium, such as immobility, dehydration, malnutrition, infections (e.g. urinary tract infection as a result of an unnecessary urinary catheter), and polypharmacy $[10,11]$.

One method of reducing delirium induced by iatrogenic risk factors is the creation of a multidisciplinary geriatric team, which focuses on optimising care for frail older patients at risk for developing delirium. These teams, often focussing on risk factors such as immobility, dehydration, malnutrition and pain, have been found to be effective in reducing delirium incidence and severity in previous studies [11-16]. Additionally, non-pharmacological interventions can be used to prevent delirium, such as the use of a clock and calendar for orientation, bringing pictures from home to provide a point of recognition for a patient, and making sure patients use their visual and/or hearing aids adequately [2]. However, these multicomponent deliriumprevention groups are often nurse-led and do not focus specifically on medication-related problems, such as polypharmacy, unnecessary medications, or harmful medication interactions, even though polypharmacy is one of the main iatrogenic risk factors for delirium in older hospitalised patients [17].

Moreover, the evaluation of these teams has always been conducted under controlled conditions in a research setting, and not in a daily-practice setting. The aims of the present study, therefore, are (1) to describe the type of changes in medication proposed as a result of the medication review and analyse the implementation of these proposed changes by the treating physician; and (2) to evaluate the benefits of the medication review on the length of delirium, length of hospital stay, in-hospital mortality, and discharge destination in older hospitalised patients who suffered delirium. We hypothesise that patients who received a medication review, and especially those patients with polypharmacy, will benefit the most, resulting in shorter duration of delirium and length of stay; that there will be less in-hospital mortality; and that patients will be discharged back home instead of to a nursing home or alternative living environment more often compared to peers who did not receive a medication review.

\section{Methods}

\subsection{Design}

A retrospective comparative cohort study using information from digital medical records was conducted at the Maastricht University Medical Centre+ (MUMC+), a 715-bed university teaching hospital in the south of the Netherlands.

\subsection{The Medication Review Process}

The MUMC + does not have a separate geriatric department, but geriatricians and geriatric nurse practitioners (GNPs) operate throughout the hospital in a liaison-consultation role for older patients (aged 70 years or over). Since 2014, all older patients admitted to the MUMC+ are screened for frailty using the Maastricht Frailty Screening Tool for Hospitalised Patients (MFST-HP) [18]. Patients with a score of eight or more on the MFST-HP are consulted by a GNP. Additionally, a consultation from a geriatrician or GNP can be requested by a nurse or physician. During these consultations delirium risk is assessed and preventive measures are discussed with the nursing staff of the patient's ward. If a patient is delirious at the time of the consultation, delirium management is discussed with the nursing staff.

In addition to these consultations, a weekly geriatric meeting was set up in February 2015, consisting of a geriatrician, a geriatrician in training, two GNPs, and a clinical pharmacist. The goal of this additional geriatric meeting was to provide a medication review for all patients who received a geriatric consult. Every week, one day before the weekly geriatric meeting, the clinical pharmacist (author LvdV) receives a list of all the patients who received a consultation from the geriatrician or GNP and were still hospitalised at the time of the weekly geriatric meeting. The clinical pharmacist then uses the STOPP (Screening Tool of Older Person's Prescriptions) and START (Screening Tool to Alert doctors to Right Treatment) criteria [19] to assess the indication, necessity, and adequacy of every medication on a patient's medication list, taking into account the age and comorbidities of the patient, as well as interactions with other medications and possible negative side effects of the medication. During the geriatric meeting the clinical pharmacist discusses the medication review with the geriatrician and GNPs, who provide extra information about the patient where needed. Based on the medication review performed by the clinical pharmacist and the discussion with the geriatrician 
and GNPs, a medication plan is formulated and the proposed changes are recorded in the patient's medical file. Figure 1 provides a flowchart of eligibility for the medication review.

\subsection{Patients}

For this study, the medical records of two patient cohorts were compared: one control cohort of patients who did not receive a medication review and a cohort of patients who did receive a medication review. The inclusion criteria for both cohorts were as follows: (1) the patients suffered delirium during their hospital stay; (2) the patients were 70 years or older on the day of admission; and (3) the patients received a consultation from either a geriatrician or GNP. Additionally, patients in the review cohort received a medication review. The control cohort consisted of the medical records of patients admitted to the MUMC+ between 1 January 2014 and 31 December 2014 (before introduction of the medication review), and the review cohort consisted of the medical records of patients admitted to the MUMC+ between 1 July 2015 and 31 December 2016 (after introduction of the medication review). The inclusion of medical records for the review cohort started 5 months after initiation of the weekly medication review meeting, to avoid start-up problems that could have influenced the study outcomes. To set up

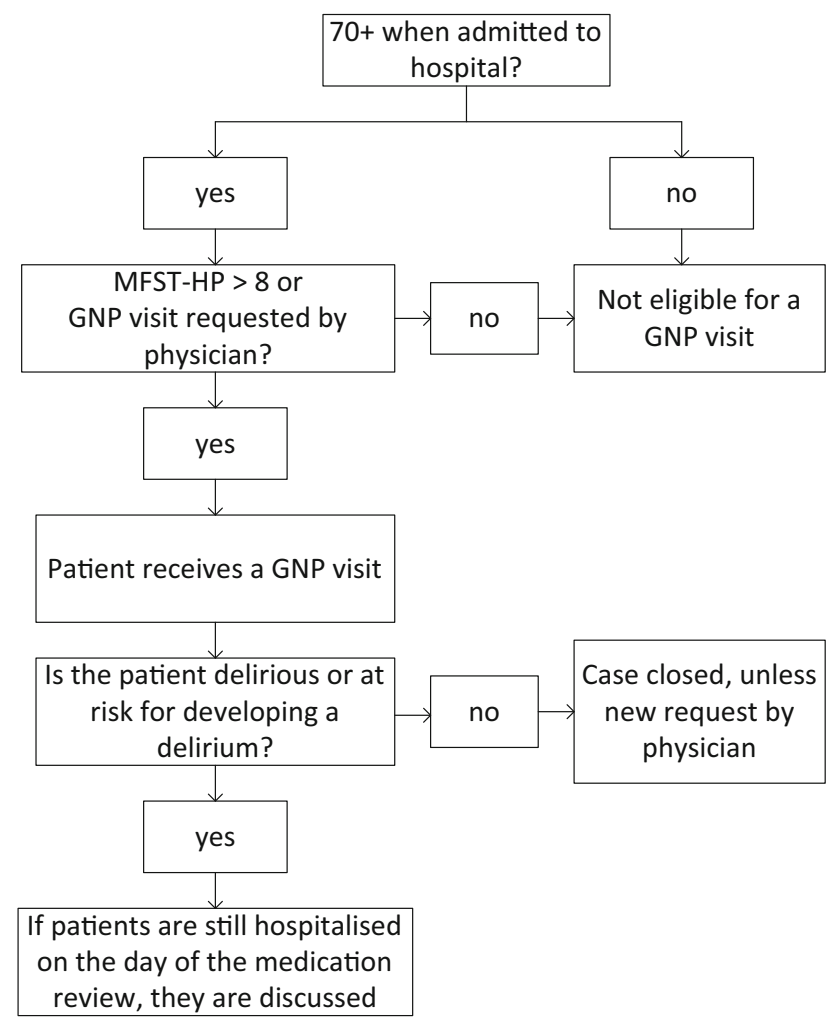

Fig. 1 Flowchart of patient eligibility for the medication review. GNP geriatric nurse practitioner, MFST-HP Maastricht Frailty Screening Tool for Hospitalised Patients comparable group numbers, the length of data collection in both cohorts varies.

\subsection{Data Extraction}

\subsubsection{Patient Data}

The following data/variables were extracted from the digital medical records by a researcher (author EvV): (1) demographic data: age at hospital admission, sex, living conditions prior to hospital admission (i.e. own home or a nursing home), and reason for admission; (2) baseline data: Charlson Comorbidity Index (CCI), and number and type of medications used at time of the GNP consultation (before the medication review); (3) the proposed changes in medication and if, and to what extent, the proposed changes had been implemented by the patient's treating physician (see Sect. 2.4.2); and (4) adverse outcomes: duration of the delirium, length of hospital stay, in-hospital mortality, and discharge destination (back home, to a nursing home, or to a rehabilitation facility).

Duration of the delirium was set as follows: the beginning of the delirium was the date on which the diagnosis of delirium was first mentioned in the digital medical record. The end date of the delirium could be determined by several factors, in decreasing order of importance: (1) when a physician or GNP noted in the medical record that the delirium was in remission or had passed; (2) when the Delirium Observation Screening (DOS) (a tool to screen for delirium and monitor its course and severity [20]) scores remained below 3 points for three consecutive measurements; (3) when the patient had died during the delirious episode; or (4) when the patient had been discharged from the hospital.

\subsubsection{Data Concerning the Medication Review}

Authors EP (geriatrician) and LvdV (clinical pharmacist) both independently and blinded to the conclusions of the other assessed all of the proposed medication changes in terms of type of change and whether or not the proposed change was implemented by the treating physician. After completion, the results of both authors were compared. In the case of a discrepancy, the patient was re-assessed by authors EP and LvdV together, until consensus was reached. The proposed changes were categorised into "start", "stop", or "change", meaning starting new medication, stopping currently used medication, or changing the dosage, administration mode, or time of administration of a medication currently used by the patient.

Analysis of the implementation of the proposed changes was done on two levels: for each unique proposed change, and per patient, as multiple changes were proposed for 
most patients. Implementation of the proposed change by the treating physician was categorised either as "yes", "no" or "unknown" (e.g. if the proposed change included medication to be given after discharge, the researchers had no access to this data). On the patient level, the implementation was assessed as "yes" if at least one of the proposed changes for a patient's medication was implemented. If none of the proposed changes were implemented, it was assessed as "no", and if this was unclear, it was assessed as "unknown". The implementation of the proposed changes on the patient level was categorised as "yes" if at least one of the proposed changes was implemented, because the authors believe this means the treating physician read and considered the proposed changes, and proceeded to implement what she/he found beneficial for the patient.

\subsection{Ethics}

The study was approved by the Medical Ethics Committee of Maastricht University/Maastricht University Medical Centre+ (\#16-4-108).

\subsection{Statistics}

Baseline characteristics for both cohorts were compared using a two-tailed $t$ test for continuous data such as age and the CCI, and a chi-square test for nominal data such as sex, living conditions and reasons for admission. The alpha for reason for admission was set to 0.01 to correct for multiple testing. A generalised linear regression model with a negative binomial distribution was used to analyse the differences between the two cohorts for duration of delirium and length of hospital stay to correct for skewness of the data. A logistic regression was used for in-hospital mortality and discharge destination. All regression models included sex, age, CCI, and number of medications used by the patients. Non-significant covariates were removed from the model stepwise. For the adverse outcomes, an interaction term for cohort and number of medications used was added, as it is expected that especially patients who take a high number of medications may benefit from the medication review. Data were analysed using SPSS version 22 (IBM Corp., Armonk, NY).

\section{Results}

\subsection{Sample Characteristics}

The inclusion criteria were fulfilled by 125 patients in the control cohort and 93 patients in the review cohort. Table 1 presents an overview of the reasons for admission of both cohorts. Patients in the review cohort were admitted more often with a hip or femur fracture than patients in the control cohort $(p=0.01)$. There were no significant differences in other reasons for admission between the two cohorts (alpha for reasons of admission was set to 0.01 to correct for multiple testing). The two cohorts were comparable with respect to age, sex, and CCI. However, patients in the control cohort used on average significantly fewer medications compared to the patients in the review cohort (eight vs ten medications, $p<0.001$ ) (see Table 2).

\subsection{Medication Review}

All 93 patients in the review cohort received a medication review by the clinical pharmacist and geriatrician. For 75 patients $(81 \%)$, changes regarding the medication were proposed, while for 18 patients (19\%) no changes were proposed. Changes were proposed on 180 accounts, with a mean of 1.9 changes per patient (range $0-7$ ). On the level of each unique proposed change, independent of the patient, most changes (62\%) were implemented by the treating physician, $32 \%$ were not implemented, and $7 \%$ were unknown. The proposed changes consisted of starting a new medication in $17 \%$, stopping certain medications in $40 \%$, and changing either the dosage, administration mode, or time of administration in $43 \%$. The majority $(40 \%)$ of the proposed changes concerned psychotropic medication, especially stopping or changing (see Table 3 for the details). The proposed changes were implemented for 58 out of the 75 patients $(77 \%)$.

\subsection{Adverse Outcomes}

The duration of delirium in the review cohort was on average 6.91 days shorter compared to the control cohort (8.56 vs 15.47 days, respectively, showing a difference of almost 50\%). A significant interaction effect between cohort and number of medications was found for the duration of delirium. Patients in the review cohort taking up to six medications had significantly shorter delirious episodes compared to patients taking up to six medications in the control cohort (mean difference 15.46 days, $p<0.001$ ). For patients taking between seven and 11 medications, the mean difference in duration of delirium between the two cohorts was 3.74 days $(p=0.07)$. For patients taking 12 or more medications, the mean difference in duration of delirium between the cohorts was 1.81 days $(p=0.55)$ (see Table 4 for the interaction effect). The other variables in the model (age, sex, CCI) were not significant and thus removed from the model. No significant differences were found for length of stay, inhospital mortality, or discharge destination. 
Table 1 Primary reasons for admission of the patients in both cohorts

\begin{tabular}{|c|c|c|c|c|}
\hline Reasons for admission & Total $(N=218)(\%)$ & Control cohort $(N=125)(\%)$ & Review cohort $(N=93)(\%)$ & $P$ value \\
\hline Cardiovascular problems & $35(16)$ & $17(14)$ & $18(19)$ & 0.25 \\
\hline Infections & $32(15)$ & $16(13)$ & $16(17)$ & 0.36 \\
\hline Hip or femur fractures & $60(28)$ & $26(21)$ & $34(37)$ & 0.01 \\
\hline General downturn & $15(7)$ & $8(6)$ & $7(8)$ & 0.75 \\
\hline Oncological causes & $8(4)$ & $6(5)$ & $2(2)$ & 0.30 \\
\hline CVA/trauma capitis & $9(4)$ & $8(6)$ & $1(1)$ & 0.05 \\
\hline Delirium or confusion & $17(8)$ & $13(10)$ & $4(4)$ & 0.10 \\
\hline Pulmonary causes (other than infections) & $9(4)$ & $9(7)$ & $0(0)$ & 0.03 \\
\hline Gastro-intestinal and intra-abdominal issues & $17(8)$ & $13(10)$ & $4(4)$ & 0.10 \\
\hline Other & $16(7)$ & $9(7)$ & $7(8)$ & 0.93 \\
\hline
\end{tabular}

Classification of the reasons for admission into the tabulated categories can be found in Electronic Supplementary Material Appendix S1

CVA cerebrovascular accident

Differences between the cohorts were calculated using a chi-square test; alpha was set to 0.01 to correct for multiple testing

Table 2 Demographic characteristics and baseline data of the total sample and of both cohorts (control and review cohorts)

\begin{tabular}{|c|c|c|c|c|}
\hline & Total $(N=218)$ & Control cohort $(N=125)$ & Review cohort $(N=93)$ & $P$ value \\
\hline Female, $n(\%)$ & $98(45 \%)$ & $58(46 \%)$ & $40(43 \%)$ & 0.62 \\
\hline Age mean, $\pm \mathrm{SD}$ (range), years & $83 \pm 6(70-98)$ & $82 \pm 6(70-97)$ & $83 \pm 8(70-98)$ & 0.67 \\
\hline Living at home before admission, $n(\%)$ & $179(82 \%)$ & $104(83 \%)$ & $75(81 \%)$ & 0.72 \\
\hline $\mathrm{CCI}$, mean $\pm \mathrm{SD}$ (range) & $7 \pm 2(3-16)$ & $7 \pm 2(3-16)$ & $7 \pm 2(3-14)$ & 0.82 \\
\hline Number of medications, mean \pm SD (range) & $9 \pm 4(0-24)$ & $8 \pm 4(1-19)$ & $10 \pm 5(0-24)$ & $<0.001$ \\
\hline
\end{tabular}

CCI Charlson Comorbidity Index, $S D$ standard deviation

Table 3 Medication advice given during the medication review categorised by type of medication, type of change, and whether or not the proposed change in medication was implemented by the physician in charge

\begin{tabular}{|c|c|c|c|c|c|c|c|}
\hline \multirow{2}{*}{$\begin{array}{l}\text { Advice provided per type of } \\
\text { medication }\end{array}$} & \multirow{2}{*}{$\begin{array}{l}\text { Number of suggested } \\
\text { changes }(N=180)(\%)\end{array}$} & \multicolumn{3}{|c|}{ Type of suggested change } & \multicolumn{3}{|c|}{ Changes implemented } \\
\hline & & Start & Stop & Change & Yes & No & Unknown \\
\hline Psychotropic & $72(40)$ & 6 & 26 & 40 & 44 & 26 & 2 \\
\hline Cardiovascular & $25(14)$ & 2 & 11 & 12 & 14 & 8 & 3 \\
\hline Analgesics and opioids & $16(9)$ & 5 & 8 & 3 & 10 & 5 & 1 \\
\hline Anti-coagulation & $15(8)$ & 3 & 2 & 10 & 8 & 7 & 0 \\
\hline Antibiotics and anti-inflammatory & $11(6)$ & 2 & 4 & 5 & 8 & 2 & 1 \\
\hline Diabetes & $6(3)$ & 2 & 3 & 1 & 4 & 2 & 0 \\
\hline Urological & $5(3)$ & 0 & 3 & 2 & 4 & 1 & 0 \\
\hline Other pharmacological advice & $30(17)$ & 11 & 15 & 4 & 19 & 6 & 5 \\
\hline Total pharmacological advice & $180(100)$ & $31(17 \%)$ & $72(40 \%)$ & $77(43 \%)$ & $111(62 \%)$ & $57(32 \%)$ & $12(7 \%)$ \\
\hline
\end{tabular}

Start starting new medication, Stop stopping used medication, Change changing the dosage, administration mode, or time of administration of a medication already used by the patient 
Table 4 Adverse outcomes for the total, control, and intervention cohorts

\begin{tabular}{|c|c|c|c|c|c|c|c|}
\hline \multirow[t]{2}{*}{ Adverse outcomes } & \multirow{2}{*}{$\begin{array}{l}\text { Total } \\
(N=218)\end{array}$} & \multirow{2}{*}{$\begin{array}{l}\text { Control cohort } \\
(N=125)\end{array}$} & \multirow{2}{*}{$\begin{array}{l}\text { Review cohort } \\
(N=93)\end{array}$} & \multirow{2}{*}{$\begin{array}{l}B, \mathrm{MD} \text { or } \\
\mathrm{OR}\end{array}$} & \multicolumn{2}{|l|}{$95 \% \mathrm{CI}$} & \multirow[t]{2}{*}{$P$ value } \\
\hline & & & & & Lower & Upper & \\
\hline $\begin{array}{l}\text { Duration of delirium in days, mean } \\
{\text { (range })^{\mathrm{a}}}^{\text {(n) }}\end{array}$ & $12.52(1-99)$ & $15.47(1-99)$ & $8.56(1-45)$ & & & & \\
\hline $\begin{array}{l}\text { Interaction group } \times \text { number of } \\
\text { medications }\end{array}$ & & & & $B=0.08$ & 0.02 & 0.15 & 0.01 \\
\hline $\begin{array}{l}\text { For patients taking } 0-6 \\
\text { medications, mean }\end{array}$ & & 20.34 & 4.88 & $\mathrm{MD}=15.46$ & 9.25 & 21.67 & $<0.001$ \\
\hline $\begin{array}{l}\text { For patients taking } 7-11 \\
\text { medications, mean }\end{array}$ & & 13.6 & 9.86 & $\mathrm{MD}=3.74$ & -0.35 & 7.83 & 0.07 \\
\hline $\begin{array}{l}\text { For patients taking } \\
12+\text { medications, mean }\end{array}$ & & 10.57 & 8.76 & $\mathrm{MD}=1.81$ & -4.15 & 7.78 & 0.55 \\
\hline $\begin{array}{l}\text { Length of stay in days, median } \\
\text { (range })^{\mathrm{a}, \mathrm{b}}\end{array}$ & $16(1-105)$ & $17(1-105)$ & $15(3-80)$ & $B=0.88$ & 0.71 & 1.09 & 0.22 \\
\hline Died in hospital, $n(\%)$ & $24(11 \%)$ & $15(12 \%)$ & $9(10 \%)$ & $\mathrm{OR}=0.74$ & 0.305 & 1.814 & 0.51 \\
\hline Discharged back home $^{\mathrm{c}}, n(\%)$ & $66(37 \%)$ & $39(38 \%)$ & $27(36 \%)$ & $\mathrm{OR}=1.01$ & 0.536 & 1.916 & 0.97 \\
\hline
\end{tabular}

All models were corrected for age, sex, Charlson Comorbidity Index, and number of medications used by the patient

$B$ unstandardised coefficient, $C I$ confidence interval, $M D$ mean difference from the generalised linear regression model, $O R$ odds ratio

${ }^{a}$ For the duration of delirium, an interaction effect between cohort and number of medications was found. To illustrate this interaction effect, the mean duration of delirium for patients taking $5,10,15$ or 20 medications for both cohorts has been included in the table

${ }^{b}$ For the length of stay, none of the covariates were significant; the table therefore displays the unadjusted model

${ }^{\mathrm{c}}$ Discharged back home was only calculated for those patients living at home before hospital admission, and therefore has an $N$ of $179(N=104$ in the control condition, and $N=75$ in the medication review condition)

\section{Discussion}

This study examined the benefit of a medication review in addition to standard geriatric care concerning delirium. Several adverse outcomes for patients with delirium (length of stay, duration of delirium, in-hospital mortality, and discharge destination) were studied. Furthermore, the medication review and proposed changes to a patient's medication were described in detail: what types of medications were subject to change, and whether or not the proposed changes were implemented by the physicians. An interaction effect of cohort and the number of medications a patient was taking was found for the duration of delirium: patients taking few medications in the review cohort had significantly shorter delirious episodes than their peers in the control cohort. This effect disappeared when the number of medications taken by a patient increased to seven or more. No significant differences were found for length of stay, in-hospital mortality, or discharge destination.

The initial hypothesis was that the medication review would benefit patients on all the negative outcomes of delirium. It was also hypothesised that this effect would be especially noticeable for patients taking more than six medications, as the chance of drug interactions and negative side effects increases with every additional drug.
However, contrary to the hypothesis, the duration of the delirium in the review cohort was reduced significantly for patients taking between zero and six medications. For patients taking between seven and 11 medications, the difference in duration of delirium bordered significance, and for patients taking twelve or more medications there was no significant difference. This indicates that patients with fewer medications might have benefitted more from the medication review. A possible explanation for this could be that the relative impact of a change decreases when the number of medications increases. Another possibility is that patients taking more medications may have more morbidities and may therefore have delirium which is caused by several factors, which may be more difficult to detect and treat.

The shorter duration of delirium for patients discussed in the review cohort is in line with previous studies that found that geriatric consultation teams focusing on risk factors such as medication and polypharmacy can prevent or shorten a delirious episode [15]. Moreover, in addition to being statistically significant, a shorter delirious episode is also highly clinically relevant: a systematic review has shown that longer or persistent delirium is associated with increased delirium severity [21] and with slower or no functional recovery [22]. One study found that functional recovery for each day of admission without delirium was 
inversely correlated with duration of the delirium (i.e. the shorter the duration of the delirium, the better the functional recovery) [23]. Better and swifter functional recovery is not only beneficial for the patient, but could also lead to lower healthcare costs as healthier patients would be more independent and less likely to be admitted to a longterm care facility or readmitted to hospital.

Despite the shorter duration of delirium among patients taking fewer medications in the intervention cohort, no effects of the medication review were found on the other outcomes (length of stay, mortality, and discharge destination). It could be argued that a shorter delirious episode leads to a faster functional recovery and thus a shorter length of hospital stay. However, we did not find any significant differences in length of hospital stay between the two cohorts in the current study. With regards to mortality, we only included data about in-hospital mortality and did not examine mortality post-discharge. Delirium can lead to increased risk of death within 12 months after the delirium [5]. It could be assumed that patients with a shorter delirious episode had lower mortality rates post discharge, but those conclusions are beyond the scope of the present study. Discharge destination was also similar in both cohorts. One may assume that patients who took fewer medications (1-6) were the cohort's healthier patients, of whom most were able to return to their previous living arrangements after hospital discharge.

In addition to the clinical effects, the costs of delirium have been estimated to be $\$ 164$ billion in the USA on a yearly basis [8]. Caring for delirious patients can be burdening for nurses [24] and may be challenging [25]. A German study showed that nurses spend an average of 240 min extra and physicians 66 min extra on the care of delirious patients compared to non-delirious controls, and the costs were estimated to be about $€ 1200$ extra per day for each patient with hyperactive delirium [9]. Taking this information into account, and considering that between 29 and $64 \%$ of older hospitalised patients suffer a delirious episode during their hospital stay [2], reducing the duration of a patient's delirium could decrease healthcare costs. Although a cost-effectiveness analysis was beyond the scope of this study, it is not improbable that this might be a cost-effective intervention, considering the shorter delirious episodes.

The majority of the changes $(40 \%)$ in medication were made for psychotropic medication, followed by cardiovascular medication (14\%). Most of the suggested changes for all types of medication were either to stop the medication or to change it; only a few changes (17\%) involved starting a new medication. This suggests that many patients take unnecessary medication, especially psychotropic medication, such as benzodiazepines, which can be highly addictive, lose effectiveness after prolonged use, and may increase the risk of falling in older people due to their sedative effects. About two thirds of all proposed changes were implemented by the treating physicians. At least one or more of the proposed changes were implemented for about $70 \%$ of the patients. This indicates that in some cases physicians may have deliberately made the choice to implement some of the proposed changes and disregard others, possibly for clinical or treatment reasons. Another reason for not implementing a proposed change may have been that the treating physician did not read the recommendations made by the review team. However, we could not uncover what the reasons were for not implementing the proposed changes.

Medication reviews are part of the national and international guidelines for the prevention and management of delirium in hospital patients. However, these guidelines are not common practice for all older patients: in our study, medication review was only done for those patients who were identified as frail using a frailty index, or patients for whom a physician requested a review. This means that many patients, including delirious patients, did (and do) not receive a medication review, despite the guidelines. The results from this study show that a medication review for all patients can be beneficial with regard to the duration of delirium. These results could be used as evidence of best practice and might help to show the added value of a hospital-wide medication review, thus creating more awareness of the importance of a medication review for all older patients. This awareness could eventually also lead to a change in prescribing practice throughout the hospital, where physicians, policy makers, other healthcare workers, and patients and family members become more aware of possible dangers of medications and their interaction with other medications.

\subsection{Limitations and Strengths}

Some issues regarding the methodology of the present study need to be considered. A retrospective cohort study was performed, instead of a randomised controlled trial (RCT). Although this choice was mainly made for practical reasons - the medication review had already been introduced at the start of the study - it could also be argued that an RCT in this case may not have been ethical, as this would mean withholding patients from care that may benefit them without causing any negative side effects. A downside to the retrospective design of the current study was that delirium diagnosis could not be standardised using a diagnostic instrument. Instead, delirium was diagnosed by two GNPs on the basis of the Diagnostic and Statistical Manual of Mental Disorders 4th edition (DSM-IV) [1] criteria for delirium. Although the GNPs are trained to diagnose delirium and patients are discussed with a 
geriatrician, the quality of the study could have been improved if delirium diagnosis was standardised beforehand. Moreover, causality cannot be implied when using a retrospective design. Furthermore, the cohorts were different in size: the control cohort was larger than the medication review cohort (125 vs 93 patients, respectively). This was because not all patients who had suffered delirium during their hospitalisation could be included in the medication review cohort. Medication reviews were only conducted on Thursdays, thus patients who were discharged back home before the medication review could take place were not included in the review cohort. Moreover, due to the retrospective nature of this study, power calculations were not performed. It would be advisable, therefore, to replicate the study in an RCT and with cohort sizes based on power calculations.

Strengths of the study are several. First, the retrospective nature of this study let us examine an intervention in daily practice, outside of a controlled research setting. Our results are therefore not influenced by a potential bias of the physicians to implement the suggested changes to the medication. It also proves that such medical interventions are viable and can be effective outside a research setting. Second, there was a relatively short period (5 months) between the end of the control cohort and the start of the review cohort. This minimises the chance that a part of the effects could be attributed to external factors, as there were no changes in national guidelines or standard procedures within the hospital during that period. Third, relevant medical records in both cohorts were identified in the same manner and had the same inclusion criteria, minimising the chances of selection bias.

\section{Conclusion}

Our findings indicate that a medication review, carried out by a clinical pharmacist and a geriatrician, may significantly decrease the duration of the delirium during hospitalisation, especially for patients taking few medications. A clinical pharmacist, therefore, is a valuable addition to a geriatric team, as they have a deep understanding of how medications work and interact, and how to best review a patient's medication list. Based on the clinical benefits of a shorter delirious episode, we recommend that a team consisting of at least a medical pharmacist and a geriatrician perform medication reviews for all older patients in a hospital. This could possibly also contribute to the prevention of delirium.

Author Contributions EV contributed to the concept and design of the study, data collection, statistical computations, and drafting of the manuscript. SZ, FV and GK contributed to the concept and design of the study, provision of clinical content, and critical revision of the manuscript. WM contributed to the concept and design of the study and provided clinical content. EP and LV contributed to data collection and critical revision of the manuscript. TA conducted the statistical analyses and contributed to the critical revision of the manuscript. All authors gave their approval for the final version of the article to be published.

\section{Compliance with Ethical Standards}

Funding No sources of funding were used to assist in the conduct of this study or the preparation of this article.

Conflict of Interest Eveline van Velthuijsen, Sandra Zwakhalen, Evelien Pijpers, Liesbeth van de Ven, Ton Ambergen, Wubbo Mulder, Frans Verhey and Gertrudis Kempen declare that they have no conflicts of interest relevant to the content of this article.

Ethical Approval Ethical approval for this study was obtained from the Medical Ethical Committee of the Maastricht University Medical Centre + . All procedures performed in studies involving human participants were in accordance with the ethical standards of the institutional and/or national research committee and with the 1964 Helsinki Declaration and its later amendments or comparable ethical standards. This is a retrospective study; for this type of study no formal consent is required.

Open Access This article is distributed under the terms of the Creative Commons Attribution-NonCommercial 4.0 International License (http://creativecommons.org/licenses/by-nc/4.0/), which permits any noncommercial use, distribution, and reproduction in any medium, provided you give appropriate credit to the original author(s) and the source, provide a link to the Creative Commons license, and indicate if changes were made.

\section{References}

1. American Psychiatric Association. Diagnostic and statistical manual of mental disorders. 4th ed. Washington, DC: American Psychiatric Association; 2000.

2. Inouye SK, Westendorp RGJ, Saczynski JS. Delirium in elderly people. Lancet. 2014;383(9920):911-22.

3. Davis DH, Muniz Terrera G, Keage H, Rahkonen T, Oinas M, Matthews FE, et al. Delirium is a strong risk factor for dementia in the oldest-old: a population-based cohort study. Brain. 2012;135(Pt 9):2809-16.

4. Krogseth M, Watne LO, Juliebo V, Skovlund E, Engedal K, Frihagen F, et al. Delirium is a risk factor for further cognitive decline in cognitively impaired hip fracture patients. Arch Gerontol Geriatr. 2016;64:38-44.

5. Witlox J, Eurelings LS, de Jonghe JF, Kalisvaart KJ, Eikelenboom P, van Gool WA. Delirium in elderly patients and the risk of postdischarge mortality, institutionalization, and dementia: a meta-analysis. JAMA. 2010;304(4):443-51.

6. Bail K, Goss J, Draper B, Berry H, Karmel R, Gibson D. The cost of hospital-acquired complications for older people with and without dementia; a retrospective cohort study. BMC Health Serv Res. 2015;15:91. https://doi.org/10.1186/s12913-015-0743-1.

7. Leslie DL, Inouye SK. The importance of delirium: economic and societal costs. J Am Geriatr Soc. 2011;59(Suppl 2):S241-3. 
8. Leslie DL, Marcantonio ER, Zhang Y, Leo-Summers L, Inouye SK. One-year health care costs associated with delirium in the elderly population. Arch Intern Med. 2008;168(1):27-32.

9. Weinrebe W, Johannsdottir E, Karaman M, Fusgen I. What does delirium cost? An economic evaluation of hyperactive delirium. Z Gerontol Geriatr. 2015. https://doi.org/10.1007/s00391-0150871-6.

10. Inouye SK, Bogardus ST, Charpentier PA, Leo-Summers L, Acampora D, Holford TR, et al. A multicomponent intervention to prevent delirium in hospitalized older patients. N Engl J Med. 1999;340(9):669-76.

11. Marcantonio ER, Flacker JM, Wright RJ, Resnick NM. Reducing delirium after hip fracture: a randomized trial. J Am Geriatr Soc. 2001;49(5):516-22.

12. Bjorkelund KB, Hommel A, Thorngren KG, Gustafson L, Larsson S, Lundberg D. Reducing delirium in elderly patients with hip fracture: a multi-factorial intervention study. Acta Anaesthesiol Scand. 2010;54(6):678-88.

13. Inouye $S$, Robinson $T$, Blaum C. Postoperative delirium in older adults: best practice statement from the American Geriatrics Society. J Am Coll Surg. 2015;220(2):136-48.

14. Milisen K, Foreman MD, Abraham IL, De Geest S, Godderis J, Vandermeulen E, et al. A nurse-led interdisciplinary intervention program for delirium in elderly hip-fracture patients. J Am Geriatr Soc. 2001;49(5):523-32.

15. Rizk P, Morris W, Oladeji P, Huo M. Review of postoperative delirium in geriatric patients undergoing hip surgery. Geriatr Orthop Surg Rehab. 2016;7(2):100-5.

16. Vidan M, Serra JA, Moreno C, Riquelme G, Ortiz J. Efficacy of a comprehensive geriatric intervention in older patients hospitalized for hip fracture: a randomized, controlled trial. J Am Geriatr Soc. 2005;53(9):1476-82.
17. Ahmed S, Leurent B, Sampson EL. Risk factors for incident delirium among older people in acute hospital medical units: a systematic review and meta-analysis. Age Ageing. 2014;43:1-8. https://doi.org/10.1093/ageing/afu022.

18. Warnier R, van Rossum E, van Leendert J, Pijls N, Mulder W, Schols J, et al. Screening for frailty in hospitalized older adults: reliability and feasibility of the Maastricht Frailty Screening Tool for Hospitalized Patients (MFST-HP). Res Gerontol Nurs. 2016;9(5):243-51.

19. Gallagher P, Ryan C, Byrne S, Kennedy J, O’Mahony D. STOPP (Screening Tool of Older Person's Prescriptions) and START (Screening Tool to Alert doctors to Right Treatment). Consensus validation. Int J Clin Pharm Ther. 2008;46(2):72-83.

20. Schuurmans MJ, Shortridge-Baggett LM, Duursma SA. The Delirium Observation Screening Scale: a screening instrument for delirium. Res Theory Nurs Pract. 2003;17(1):31-50.

21. Dasgupta M, Hillier LM. Factors associated with prolonged delirium: a systematic review. Int Psychogeriatr. 2010;22(3):373-94.

22. Kiely DK, Jones RN, Bergmann MA, Murphy KM, Orav EJ, Marcantonio ER. Association between delirium resolution and functional recovery among newly admitted postacute facility patients. J Gerontol A Biol Sci Med Sci. 2006;61(2):204-8.

23. Speciale S, Bellelli G, Lucchi E, Trabucchi M. Delirium and functional recovery in elderly patients. J Gerontol A Biol Sci Med Sci. 2007;62(1):107-8 (author reply 8).

24. Mc Donnell S, Timmins F. A quantitative exploration of the subjective burden experienced by nurses when caring for patients with delirium. J Clin Nurs. 2012;21(17-18):2488-98.

25. Day J, Higgins I, Keatinge D. Orientation strategies during delirium: are they helpful? J Clin Nurs. 2011;20(23-24):3285-94. 